\title{
Using circular dichroism to control energy transfer in multi-photon ionization
}

\author{
A.H.N.C. De Silva,${ }^{1}$ D. Atri-Schuller,${ }^{2}$ S. Dubey,${ }^{1}$ B.P. Acharya,${ }^{1}$ K.L. Romans,${ }^{1}$ K. Foster,${ }^{1}$ \\ O. Russ,${ }^{1}$ K. Compton, ${ }^{1}$ C. Rischbieter, ${ }^{1}$ N. Douguet,${ }^{3}$ K. Bartschat,${ }^{2}$ and D. Fischer ${ }^{1}$ \\ ${ }^{1}$ Physics Department and LAMOR, Missouri University of Science 83 Technology, Rolla, MO 65409, USA \\ ${ }^{2}$ Department of Physics and Astronomy, Drake University, Des Moines, Iowa 50311, USA \\ ${ }^{3}$ Department of Physics, Kennesaw State University, Kennesaw, Georgia 30144, USA
}

(Dated: January 13, 2021)

\begin{abstract}
Chirality causes symmetry breaks in a large variety of natural phenomena ranging from particle physics to biochemistry. We investigate one of the simplest conceivable chiral systems, a laserexcited, oriented, effective one-electron Li target. Prepared in a polarized $p$ state with $|m|=1$ in an optical trap, the atoms are exposed to co- and counter-rotating circularly polarized femtosecond laser pulses. For a field frequency near the excitation energy of the oriented initial state, a strong circular dichroism is observed and the photoelectron energies are significantly affected by the helicitydependent Autler-Townes splitting. Besides its fundamental relevance, this system is suited to create spin-polarized electron pulses with a reversible switch on a femtosecond timescale at an energy resolution of a few meV.
\end{abstract}

Circularly polarized light exhibits handedness, a feature that gives rise to symmetry breaks in its interaction with matter. This intriguing phenomenon is well known as circular dichroism (CD), and it unfolds, e.g., as the difference in photoelectron angular distributions (PADs) for opposite photon helicities in single-photon ionization of oriented diatomic molecules [1] and even of groundstate atomic targets [2]5]. For these nonchiral targets, however, the systems of opposite photon handedness are merely mirror images of one another (neglecting parityviolating effects [6, 7]). This mirror symmetry is only lifted, therefore, if the target also possesses a handedness. Chiral molecules, i.e., molecules that are not superimposable with their mirror images, are a prominent example of such handed targets. Their ionization by single- 8 or multi-photon [11]13] absorption as well as strong optical fields [14] can reveal significant dichroic asymmetries even for randomly oriented molecules. Such asymmetric photoreactions have far-reaching implications that could contribute to the solution of the long-standing puzzle of the homochirality of amino acids and sugar molecules, which are relevant for terrestrial life [15, 16].

Single atoms can also feature chirality if their orbital angular momentum is polarized along the projectile beam direction with a mean magnetic quantum number $\langle m\rangle \neq 0$ [17. In contrast to chiral molecules, these systems are still superimposable with their mirror images, i.e., they do not have an intrinsic chirality. However, the atoms' helicity combined with an external anisotropy, e.g. given by the direction of an incoming photon's spin, results in a handedness, which sometimes is referred to as "external chirality" [17. Due to their comparably simple structure, polarized atomic targets represent benchmark systems for our understanding of asymmetries in the interaction of chiral light with chiral matter.

Recent studies of atomic dichroic effects focussed, amongst other things, on fundamental aspects of magneto-optics [18] or on the details of tunneling dynam- ics 1921 and resonance-enhanced multi-photon ionization (REMPI) [17, 22, 23]. Circular dichroism is typically stronger in polarized atoms than in chiral molecules, because it occurs already in the electric dipole approximation while molecular targets require generally magnetic (or, for oriented molecules, higher-order electric) contributions to expose asymmetries (e.g. 24]). For electric dipole transitions, the magnetic quantum number $m$ changes by +1 or -1 for each absorbed photon of rightor left-handed circular polarization, respectively. Consequently, the helicity dependence in ionization of polarized atoms can be explained in terms of different partial waves contributing and interfering in the final state [25, 26], resulting in dichroic asymmetries in total ionization yields and PADs. Dichroic shifts in the photoelectron energies, in contrast, are either completely absent [26] or relatively small (as compared to peak widths and positions), but they can give insights into the structure of the dressed target atoms 22, 23, 27] or reveal fingerprints of atomic ring currents $[20,28]$.

In this Letter, we demonstrate an atomic multi-photon ionization scheme, in which circular dichroism manifests itself in strong and controllable shifts in the photoelectron energy spectrum. Alkali atoms are optically pumped to a polarized $p$-state and subsequently ionized by the absorption of two photons in the circularly polarized field of an intense femtosecond laser. A change of the relative helicity of atoms and field results in shifts of the photoelectron energies by up to $40 \%$ (about $100 \mathrm{meV}$ ). This observation is qualitatively understood by the polarization-selective Autler-Townes splitting of the initial state due to its coupling to the ground state in the intense light field. The counter-intuitive energy dependence on the photons' polarization adds a new dimension to dichroic phenomena in photon-atom interactions and provides an additional dial for the quantum control of the emission of polarized electrons [29, 30]. It can also be used to enhance the chiral response in the analysis of 
handed targets.

The present study contributes to the interesting and much-debated question whether photoionization proceeds more efficiently for the electron current density of the initial state being co-rotating or counterrotating with the ionizing field. For low-intensity singlephoton absorption, it is well-established that ionization is strongly favored in the co-rotating case 25, but the trend was found to be reversed in the nonadiabatic tunnel ionization regime [19, 28. For multi-photon ionization, in contrast, this question was not answered unambiguously. It was found that the favored geometry swaps with increasing field intensity [22, 31]. In these studies, intermediate excited states play an important role and the observed intensity dependence can be very strong 23] due to transient ("Freeman") resonances [32, where quasi-energies of dressed intermediate states are moved in resonance, thereby enhancing specific REMPI channels. For the present system, in contrast, intermediate excited states and resonance enhancement do not substantially affect the ionization process. Consequently, our setup represents a particularly clean manifestation of dichroic asymmetries, and it bridges the gap between the singlephoton and the strong field regimes, where the details of the target structure sway the helicity dependence of the ionization yield only marginally.

In our experiment, an atomic target gas cloud was prepared in a near-resonant all-optical laser atom trap (AOT) 33, where lithium atoms are cooled to temperatures of about $1 \mathrm{mK}$. The wavelength of the AOT laser field is tuned near the $2 s-2 p$ resonance at $671 \mathrm{~nm}$ $\left(\Delta \nu_{\mathrm{AOT}}<15 \mathrm{MHz}\right)$, resulting in a steady-state atomic excitation fraction of about $25 \%$. As shown earlier 33, optical pumping in the AOT results in a high degree of atomic polarization, with $93 \%$ of the excited state atoms populating a single magnetic sub-level with $|m|=1$. The femtosecond light source is a commercially available few-cycle optical parametric chirped-pulse amplifier (OPCPA) system similar to the setup described in 34. It is based on a Ti:Sa oscillator providing the seed for two non-collinear optical parametric amplifier (NOPA) stages. For the present experiment, the system was configured to emit pulses with a wavelength, duration, and repetition rate of $665 \pm 5 \mathrm{~nm}, 65 \mathrm{fs}$, and $200 \mathrm{kHz}$, respectively, and a peak power of up to $10^{12} \mathrm{~W} / \mathrm{cm}^{2}$. The femtosecond laser beam is focused and guided through the vacuum chamber with a waist of $50 \mu \mathrm{m}$ at the target position and an angle of $10^{\circ}$ with respect to the polarization direction of the atoms (i.e. the $z$-axis). Electron and recoil ion momenta are measured in coincidence in a cold-target recoil-ion momentum spectrometer (COLTRIMS) 35.

The experimental data shown below are compared with predictions from an ab initio calculation based on the solution of the time-dependent Schrödinger equation (TDSE). For the setup considered here, lithium can be

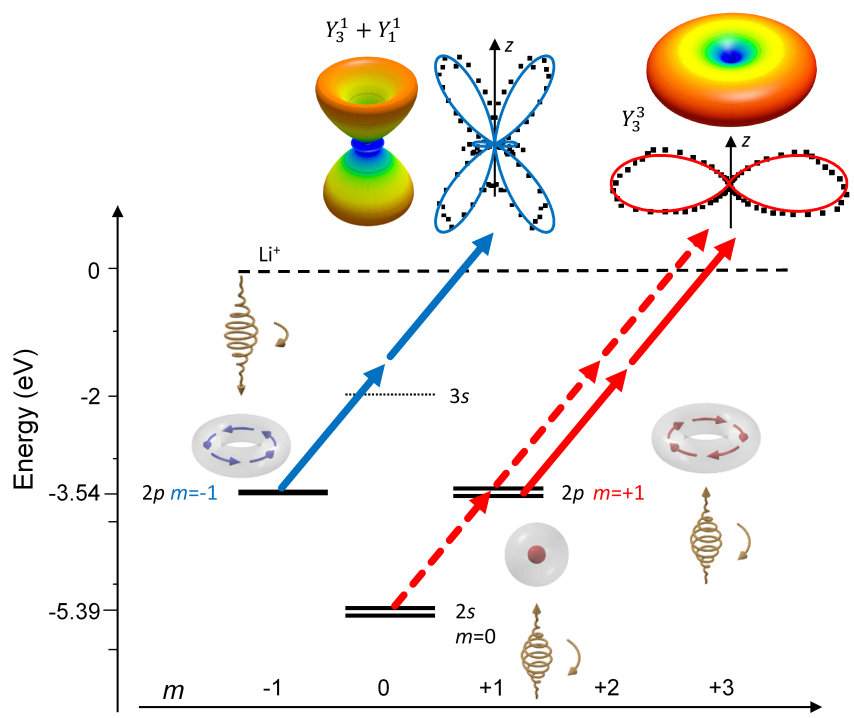

FIG. 1. Scheme of few-photon ionization of $\operatorname{Li}(2 p)$ and $\operatorname{Li}(2 s)$ in lowest-order perturbation theory. The magnetic quantum number $m$ is denoted with respect to the direction of the photon spin. Atomic levels undergoing Autler-Townes splitting are shown as double lines in the graph (see text). Measured PADs and fitted spherical harmonics are shown on the top for the counter- (left) and co-rotating case (right).

well described as an active $(n \ell)$ valence electron above an inert He-like $\left(1 s^{2}\right)$ core. The latter was simulated by the static Hartree potential supplemented by phenomenological terms to simulate the core polarizability as well as exchange between the valence electron and the core. The ideas of the method were described in [36, 37. and successfully used by Schuricke et al. 38. With a few further improvements, we obtained the ionization potentials of the $2 s$ and $2 p$ orbitals, as well as those of the $n=3$ orbitals, to better than $1 \mathrm{meV}$ of the recommended data 39]. The initial state was then propagated in time by solving the TDSE numerically [40, 41]. We used an updated version of the code with the necessary modifications introduced for circularly polarized light described by Douguet et al. 42]. The TDSE was solved in the velocity gauge and we employed a non-uniform radial grid, decreasing in the density of points from the region close to the nucleus (mesh $\Delta r=0.01$ a.u.) to the far region where we only need to describe slow electrons in this experiment (maximum mesh $\Delta r=0.5$ a.u.). The time step and maximum angular momentum used for the highest laser intensity were equal to $\Delta t=0.02 \mathrm{a}$.u. and $\ell_{\max }=12$, respectively. Finally, we have checked that our results are converged to high accuracy.

For the conditions described above, the corresponding Keldysh paramaters are always larger than about 6.5 and the ionization process is well described in a multi-photon picture. The ionization pathways are depicted in Fig. 1 according to lowest-order perturbation theory. Lithium atoms in the $2 s$ ground state are ionized by the absorp- 

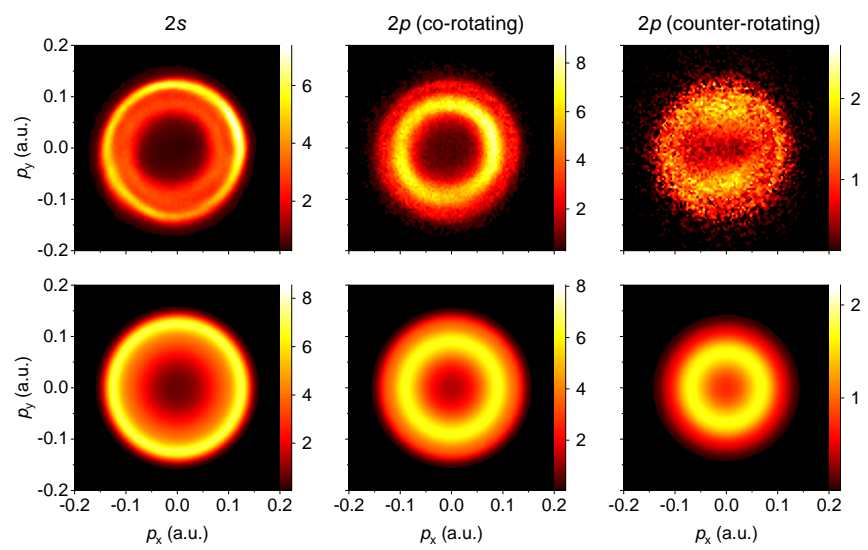

FIG. 2. Measured (top row) and calculated (bottom row) electron momentum distributions in the $x y$-plane integrated over the $z$-component for a laser field peak intensity of $0.68 \times$ $10^{12} \mathrm{~W} / \mathrm{cm}^{2}$. The initial target states are $2 \mathrm{~s}$ (left column) and $2 p$ for co-rotating (center) and counter-rotating (right) circular field polarizations.

tion of three photons, resulting in a final-state (orbital) angular momentum of $(\ell, m)=(3,3)$. Note that the center frequency of the laser pulses is near the $2 s-2 p$ zerofield resonance energy with a blue-shift of $\Delta \nu_{\mathrm{fs}} \approx 4 \mathrm{THz}$ $(16 \mathrm{meV})$. For target atoms in the excited $2 p$ state, two photons suffice to promote the valence electron to the continuum. For the co-rotating case, the final angular momentum is identical to $2 s$ ionization and hence given by $(3,3)$. For the counter-rotating case, on the other hand, the final magnetic quantum number is $m=1$, with the total angular momentum in a superposition of $\ell=1$ and $\ell=3$. The different angular momenta result in vastly different PADs, as illustrated in Fig. 1.

Figure 2 exhibits momentum distributions of lowenergy electrons in the plane perpendicular to the laser beam propagation direction (the $x y$-plane) for $2 s$ ionization as well as for co- and counter-rotating $2 p$ ionization. All spectra feature ring structures due to the cylindrical symmetry of the systems. The diameters of the rings reflect different electron continuum energies. In order to account for the spatial laser field intensity distribution in the reaction volume around the focal point, the theoretical spectra are not calculated for a single intensity, but are weighted averages [38] covering an intensity range of more than one order of magnitude with a maximum peak intensity of $0.68 \times 10^{12} \mathrm{~W} / \mathrm{cm}^{2}$. Additionally, the theoretical spectra were convolved over the experimental energy resolution of $30 \mathrm{meV}$. This procedure yields excellent agreement with the experimental spectra. The statistical quality of the experimental data for the counter-rotating case is worse than for the other two situations, essentially because a relatively small amount of data for the $2 p$ ionization is obtained by subtracting a large "background" from the $2 s$ initial state, which constitutes about $75 \%$ of the total target density.
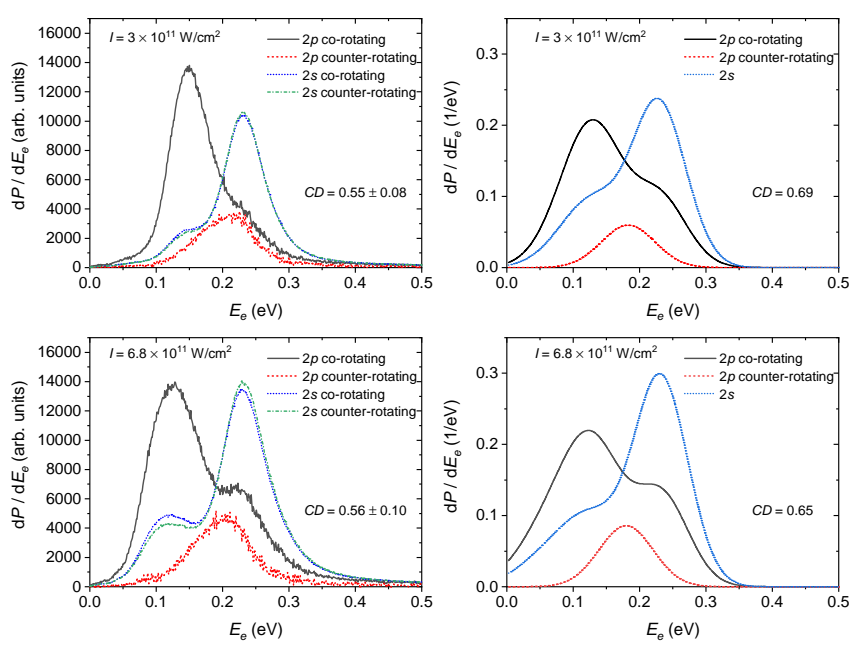

FIG. 3. Measured (left) and calculated (right) electron energy spectra for $2 s$ and $2 p$ ionization for both relative helicities of the circularly polarized laser field. Peak intensities $I$ and $C D$ values are given in the graphs.

The differential ionization probabilities as a function of the photoelectron energy are plotted in Fig. 3. The energy range shown in the figure contains more than $98 \%$ of the theoretical cross sections, i.e. contributions of higher electron energies due to above-threshold ionization (ATI) are small for the investigated field intensities. For ionization of the $2 s$ ground state, the distributions for co- and counter-rotating polarization are expected to be identical (neglecting the spin polarization of the target atoms), which is consistent with the experimental observations. Remaining deviations for the two laser polarizations are attributed to systematic uncertainties, such as small drifts in the laser spectrum or slightly different residual ellipticities of the laser polarization in the two measurements. At the lower laser intensity (top row in Fig. 3) and for ground-state ionization, the photoelectron energy peak features a shoulder towards lower electron energies, which develops into a separate maximum with increasing laser intensity. A similar behavior is observed for the $2 p$-state ionization for the co-rotating laser polarization, with the shoulder being towards the high-energy side of the main energy peak. In the counter-rotating situation, however, there is a single peak, whose position and general shape do not significantly vary with the laser intensity.

The general features of the experimental spectra are very well reproduced by our calculation, which again has been averaged and convolved according to the method discussed above. However, some differences between experiment and theory persist, in particular with respect to the relative intensities of the lines. On the theoretical side, deviations caused by model-related approximations as well as numerical uncertainties of the calculation are expected to be very small. Therefore, remaining discrep- 
ancies are more likely due to the many uncertainties in the experimental parameters (e.g. spectrum, spatial as well as temporal intensity distribution, and polarization of the laser pulses, or the polarization and population ratios of the target gas cloud), which are challenging to characterize precisely.

The structure and shifts observed in the energy spectra for $2 s$ ionization and for $2 p$ ionization with corotating polarization can be qualitatively interpreted by the "dressing" of the initial states in the photon field. The dressed-state approach is widely used to explain structures in photoelectron energy spectra for multiphoton ionization of atoms and molecules (e.g. 223, 43]) and provides intuitive insights into the physical mechanisms at play. Particularly interesting is the situation where the photon field is at resonance and couples two atomic levels. The coupling splits the levels into AutlerTownes doublets that are well known in atomic spectroscopy [44] and multi-photon ionization [45, 46]. In the dressed-atom description, these doublets stem from avoided crossings of the combined "atom + photons" states (or Floquet states) at the resonant field frequency (e.g. [4]). Their separation depends on the strength of the coupling, i.e., on the intensity of the coupling field as well as the dipole moment of the atomic transition.

In the present system, the field frequency is close to the $2 s-2 p$ resonance with a slight blue shift, splitting the two levels into doublets, which materialize as two lines (or one line with a shoulder) in the photoelectron energy distributions. For the counter-rotating field, the $2 p$ state does not exhibit the Autler-Townes splitting, because the excited initial state is not coupled to the ground state by radiation of opposite helicity (see Fig. 1). Generally, the evolution of dressed states in a femtosecond laser pulse is a time-dependent problem. Assuming the rather unrealistic case of a fully adiabatic evolution of the dressing, the blue-shifted radiation would cause an up-shift (downshift) in energy of the initially undressed $2 s(2 p)$ state, i.e., only one level out of the respective doublet would be populated. Therefore, the observation of both lines of the doublets might generally indicate the nonadiabaticity of the process with their relative intensities even providing a quantitative measure. Additionally, the electron energies are subject to ponderomotive shifts. These are, however, rather small $(\leq 21 \mathrm{meV})$ for the present field intensities.

Quantitatively, circular dichroism is given by the difference of the relative ionization yields for the two photon spins. It is defined as $C D=\left(P_{+}-P_{-}\right) /\left(P_{+}+P_{-}\right)$, where $P_{+}$and $P_{-}$are the ionization probabilities for co- $(+)$or counter-rotating $(-)$ helicities, respectively. For the two peak intensities shown in Fig. 3, the measured angleand energy-integrated $C D$ values are $0.55 \pm 0.08$ and $0.56 \pm 0.10$, respectively. Both values are in good agreement with the theoretical predictions of 0.69 and 0.65 , respectively. However, the measured values have significant uncertainties, which are dominated by the imprecise knowledge of the excited-state fraction of the atomic gas cloud $(25 \pm 3 \%)$. Contributions due to statistical errors and the cross-normalization procedure of the spectra for co- and counter-rotating radiation are relatively small. The given errors do not account for systematic effects due to the imperfect polarization of target and laser field, which generally are expected to shift the absolute $C D$ value slightly down.

It is interesting to compare the present results to other recent studies of circular dichroism in multi-photon ionization of other atomic systems. Specifically, Ilchen et al. 22 and Grum-Grzhimailo et al. 23 investigated circular dichroism in the double ionization of helium in an XUVIR two-frequency field. In this system, the absorption of two XUV photons results in the sequential and resonant ionization-excitation of the target to the polarized $\mathrm{He}^{+}(3 p)$ state, which is subsequently ionized by the absorption of four or more IR photons. Here, the integrated $C D$ value is close to $+100 \%$ at low intensities before it drops and even changes sign for higher intensities. Interestingly, this change occurs over a very narrow intensity range. Doubling the intensity already suffices to bring the $C D$ value down to nearly zero.

For the present system, the overall intensity dependence of the dichroism is much weaker and appears to be more consistent with an earlier theoretical study considering state-prepared atomic hydrogen [31. This different behavior can be understood by the vastly unalike preparation methods of the polarized $p$ states: In [22], the target excitation and the multi-photon ionization processes occur both on the same time scale in a twofrequency femtosecond radiation pulse. The steep drop of the dichroism is explained by the polarization-selective dynamic Stark shift of the $\mathrm{He}^{+}(3 p)$ state in the intense IR field shifting the XUV field and the excited target state out of resonance [23. In the present study, in contrast, the target excitation and the multiphoton ionization processes are largely disentangled, as the lithium atoms are excited in the quasi-continuous, low-intensity (in the order of $10^{-2} \mathrm{~W} / \mathrm{cm}^{2}$ ) resonant field of the AOT cooling lasers on a much longer time scale given by the lifetime of the excited state (about $27 \mathrm{~ns}$ ). Therefore, dynamic Stark shifts of the excited $2 p$ state in the femtosecond laser pulse do not significantly hamper the efficiency of the state preparation.

In conclusion, we calculated and demonstrated experimentally a multi-photon ionization scheme where strong circular dichroism occurs in the photoelectron energy distribution. Specifically, polarized atomic lithium in the excited $2 p$ state is ionized by intense circularly polarized radiation of both relative helicities with a frequency near the $2 p$ excitation energy. If the laser electric field and the target electron current density counter-rotate in the same plane, the photoelectron energy spectrum exhibits a single peak at about $2 \hbar \omega-I_{P}-U_{P}$ following simple energy conservation, with $\omega, I_{P}$, and $U_{P}$ being the field 
frequency, the ionization potential of the excited initial state, and the ponderomotive energy shift, respectively. For the co-rotating case, in contrast, this energy relation is violated, because the $2 p$ initial state and the $2 s$ ground state are coupled by the laser field, thus resulting in the Autler-Townes splitting of both states. This effect enables to control photoelectron energies by the field's intensity and polarization and induce shifts that, in the current experiment, amount up to $40 \%$ of the average continuum energy.

The multi-photon ionization scheme discussed in this Letter is ideally suited and directly applicable to create spin-polarized electron beams. It has been shown earlier that photoelectrons have a nonvanishing spin polarization depending on their continuum energy in multiphoton [30] or strong-field [29] ionization of noble-gas atoms by circularly polarized light. Due to the state preparation of the target by optical pumping in the present scheme, not only the orbital angular momentum but also the spin of the single valence electron are aligned in the initial state 33 . Therefore, a nearly complete spin polarization of the photoelectrons can be expected, irrespective of their final energy. The polarization-dependent energy shift discussed above provides an extremely fast, femtosecond switchable dial to control the electron energy on a level of a few meV. This way, femtosecond spin-polarized electron pulses can be created with applications in electron diffraction experiments probing, e.g., ultrafast spin dynamics of magnetic domains.

The experimental material presented here is based upon work supported by the National Science Foundation under Grant No. PHY-1554776. We thank Thomas Binhammer for very helpful advice about the tunability of the laser spectrum. The theoretical part of this work was funded by the NSF under Grants No. PHY1803844 (D.A.S., K.B.) and No. PHY-2012078 (N.D.), and the XSEDE supercomputer allocation No. PHY090031. The calculations were carried out on Comet at the San Diego Supercomputer Center.

[1] T. Jahnke et al., Phys. Rev. Lett. 88, 073002 (2002).

[2] J. Berakdar and H. Klar, Phys. Rev. Lett. 69, 1175 (1992)

[3] V. Mergel et al., Phys. Rev. Lett. 80, 5301 (1998)

[4] J. M. Feagin, Phys. Rev. Lett. 88, 043001 (2002).

[5] J. Hofbrucker, A. V. Volotka, and S. Fritzsche, Phys. Rev. Lett. 121, 053401 (2018)

[6] C. E. Loving and P. G. H. Sandars, J. Phys. B 8, L336 (1975)

[7] P. Bucksbaum, E. Commins, and L. Hunter, Phys. Rev. Lett. 46, 640 (1981).

[8] N. Böwering, T. Lischke, B. Schmidtke, N. Müller, T. Khalil, and U. Heinzmann, Phys. Rev. Lett. 86, 1187 (2001).
[9] G. A. Garcia, L. Nahon, S. Daly, and I. Powis, Nature Communications 4, 2132 (2013)

[10] L. Nahon, G. A. Garcia, and I. Powis, Journal of Electron Spectroscopy and Related Phenomena 204, 322 (2015), gas phase spectroscopic and dynamical studies at Free-Electron Lasers and other short wavelength sources.

[11] R. E. Goetz, C. P. Koch, and L. Greenman, Phys. Rev. Lett. 122, 013204 (2019)

12] C. Lux, M. Wollenhaupt, T. Bolze, Q. Liang, J. Köhler, C. Sarpe, and T. Baumert, Angewandte Chemie International Edition 51, 5001 (2012)

13] C. Lux, A. Senftleben, C. Sarpe, M. Wollenhaupt, and T. Baumert, J. Phys. B 49, 02LT01 (2015).

[14] S. Beaulieu et al., Nature Physics 14, 484 (2018).

[15] A. Jorissen and C. Cerf, Origins of life and evolution of the biosphere 32, 129 (2002).

[16] L. Nahon, G. Garcia, I. Powis, U. Meierhenrich, and A. Brack, in Instruments, Methods, and Missions for Astrobiology $X$. Vol. 6694, edited by R. B. Hoover, G. V. Levin, A. Y. Rozanov, and P. C. W. Davies, International Society for Optics and Photonics (SPIE, 2007) pp. $19-34$.

[17] T. Mazza et al., Nature Communications 5, 3648 (2014).

[18] J. M. Choi, J. M. Kim, Q.-H. Park, and D. Cho, Phys. Rev. A 75, 013815 (2007).

[19] T. Herath, L. Yan, S. K. Lee, and W. Li, Phys. Rev. Lett. 109, 043004 (2012).

[20] S. Eckart et al., Nature Physics 14, 701 (2018).

[21] U. S. Sainadh et al., Nature 568, 75 (2019)

[22] M. Ilchen et al., Phys. Rev. Lett. 118, 013002 (2017).

[23] A. N. Grum-Grzhimailo, N. Douguet, M. Meyer, and K. Bartschat, Phys. Rev. A 100, 033404 (2019).

[24] N. Berova, P. L. Polavarapu, K. Nakanishi, and R. W. Woody, eds., Comprehensive Chiroptical Spectroscopy (Wiley, 2014).

[25] H. A. Bethe and E. E. Salpeter, Quantum mechanics of one and two electron atoms (Plenum, New York, 1977).

[26] F. Thini et al., J. Phys. B 53, 095201 (2020).

[27] A. K. Kazansky, A. V. Grigorieva, and N. M. Kabachnik, Phys. Rev. Lett. 107, 253002 (2011).

[28] I. Barth and O. Smirnova, Phys. Rev. A 84, 063415 (2011).

[29] A. Hartung et al., Nature Photonics 10, 526 (2016).

[30] M.-M. Liu, Y. Shao, M. Han, P. Ge, Y. Deng, C. Wu, Q. Gong, and Y. Liu, Phys. Rev. Lett. 120, 043201 (2018).

[31] J. H. Bauer, F. Mota-Furtado, P. F. O'Mahony, B. Piraux, and K. Warda, Phys. Rev. A 90, 063402 (2014).

[32] R. R. Freeman, P. H. Bucksbaum, H. Milchberg, S. Darack, D. Schumacher, and M. E. Geusic, Phys. Rev. Lett. 59, 1092 (1987).

[33] S. Sharma, B. P. Acharya, A. H. N. C. D. Silva, N. W. Parris, B. J. Ramsey, K. L. Romans, A. Dorn, V. L. B. de Jesus, and D. Fischer, Phys. Rev. A 97, 043427 (2018).

[34] A. Harth, C. Guo, Y.-C. Cheng, A. Losquin, M. Miranda, S. Mikaelsson, C. M. Heyl, O. Prochnow, J. Ahrens, U. Morgner, A. L'Huillier, and C. L. Arnold, Journal of Optics 20, 014007 (2017).

[35] R. Hubele et al., Review of Scientific Instruments 86, 033105 (2015).

[36] B. J. Albright, K. Bartschat, and P. R. Flicek, J. Phys. B 26, 337 (1993)

[37] K. Bartschat, "Core Potentials for Quasi One-Electron 
Systems," in Computational Atomic Physics: Electron and Positron Collisions with Atoms and Ions, edited by K. Bartschat (Springer Berlin Heidelberg, 1996) pp. 1526.

[38] M. Schuricke et al., Phys. Rev. A 83, 023413 (2011).

[39] A. Kramida, Yu. Ralchenko, J. Reader, and NIST ASD Team, NIST Atomic Spectra Database (ver. 5.7.1), [Online]. Available: http://physics.nist.gov/asd [2020, May 10]. National Institute of Standards and Technology, Gaithersburg, MD. (2020).

[40] A. N. Grum-Grzhimailo, A. D. Kondorskiy, and K. Bartschat, J. Phys. B 39, 4659 (2006).

[41] A. N. Grum-Grzhimailo, B. Abeln, K. Bartschat, D. Weflen, and T. Urness, Phys. Rev. A 81, 043408 (2010).

[42] N. Douguet, A. N. Grum-Grzhimailo, E. V. Gryzlova,
E. I. Staroselskaya, J. Venzke, and K. Bartschat, Phys. Rev. A 93, 033402 (2016).

[43] D. I. Duncan, J. G. Story, and T. F. Gallagher, Phys. Rev. A 52, 2209 (1995).

[44] S. H. Autler and C. H. Townes, Phys. Rev. 100, 703 (1955).

[45] Z. Sun and N. Lou, Phys. Rev. Lett. 91, 023002 (2003).

[46] M. Wollenhaupt, A. Präkelt, C. Sarpe-Tudoran, D. Liese, and T. Baumert, Journal of Optics B: Quantum and Semiclassical Optics 7, S270 (2005).

[47] C. N. Cohen-Tannoudji, "The Autler-Townes effect revisited," in Amazing Light: A Volume Dedicated To Charles Hard Townes On His 80th Birthday, edited by R. Y. Chiao (Springer New York, New York, NY, 1996) pp. 109-123. 